\title{
A vacina contra o papilomavírus humano
}

\author{
Comentário sobre o artigo do Dr. Juan Gérvas \\ José Eluf Neto* \\ Departamento de Medicina Preventiva da Faculdade de Medicina - Universidade de São Paulo
}

O câncer de colo uterino é um importante problema de saúde no mundo, especialmente nos países em desenvolvimento. Estudos epidemiológicos e laboratoriais indicam a infecção persistente por papilomavírus humano (HPV) como causa necessária desse câncer ${ }^{1}$. Sabe-se atualmente que quase todos os casos de neoplasia cervical são causados pela infecção persistente com certos tipos ("alto risco") de $\mathrm{HPV}^{2}$. Estima-se que pelo menos $95 \%$ desses casos são decorrentes de infecção com cerca de 15 tipos de HPV de alto risco, sendo $70 \%$ pelos tipos 16 e $18^{3}$. Esse conhecimento contribuiu para o desenvolvimento de novas estratégias para a prevenção da doença, a mais promissora representada por vacinas profiláticas contra o HPV.

Duas vacinas foram desenvolvidas: quadrivalente (Merck) contra HPV 6, 11, 16 e 18, e bivalente (GSK) contra HPV 16 e 18. Os tipos de HPV 6 e 11 ("baixo risco") estão associados a condiloma acuminado, não a câncer cervical. Ambas as vacinas apresentam eficácia elevada, tanto na prevenção de infecção persistente com HPV 16 ou 18, quanto na prevenção de neoplasia intraepitelial cervical (NIC) 2 e $3^{4,5}$. A vacina quadrivalente foi aprovada em diversos países, inclusive no Brasil, enquanto a segunda foi aprovada na Austrália ${ }^{2}$.

Apesar dos resultados favoráveis encontrados em vários estudos, há questões e dúvidas importantes apontadas pelo Dr. Gérvas, para a introdução da vacinação contra HPV:

1. Os dois tipos de HPV de "alto risco" (16 e 18), incluídos nas duas vacinas, são responsáveis por cerca de $70 \%$ dos casos de câncer de colo de útero; portanto, se a vacina não induzir imunidade "cruzada", não haverá proteção para $30 \%$ dos casos (associados a outros tipos de HPV).

2. A eficácia da vacina foi evidenciada com resultados "intermediários" - diminuição de infecção persistente e NIC, não "finais" - diminuição de câncer cervical.

3. A duração da eficácia vacinal demonstrada até o momento é de cinco anos.

4. $O$ alto custo da vacina.

A introdução da vacina, ou a expectativa de sua introdução em futuro próximo, pode ter uma grave conseqüência, não comentada pelo Dr. Gérvas: a redução do gasto com rastreamento para câncer de colo de útero, diminuindo a cobertura ${ }^{6}$. Há várias razões para a continuidade do rastreamento dessa neoplasia por longo tempo após a introdução da vacina. A idade recomendada para vacinação é 11-12 anos, podendo ser administrada em mulheres desde nove até 26 anos de idade ${ }^{7}$. Portanto, as mulheres com maior risco de câncer cervical (idade igual ou superior a 40 anos) não estarão protegidas nos próximos anos/décadas. Outra razão é a não proteção, ou proteção limitada, contra os tipos de HPV de alto risco não incluídos na vacina. Além disso, é provável que a proteção diminua com o tempo ${ }^{4}$.

Por outro lado, o texto contém diversas afirmações questionáveis, mencionadas a seguir.

É pouco provável que se disponha de dados confiáveis sobre incidência de carci-

*Conflito de interesse: Consultoria para a GlaxoSmithKline (evento realizado em 2006). 
noma in situ no Haiti. Este país tem a maior taxa de infecção por HPV do mundo?

A citologia cervical é o exame de rastreamento menos avaliado cientificamente (por falta de ensaios clínicos controlados)?

Em mulheres entre 30 e 65 anos de idade, com três citologias consecutivas normais, a incidência de câncer cervical é zero?

Não entendi a crítica do Dr. Gérvas à via de administração (intramuscular) da vacina contra HPV. A maioria das vacinas é administrada por injeção intramuscular, independente da forma de transmissão do agente infeccioso.

Como seria possível mostrar a eficácia da vacina no grupo de crianças do sexo feminino, com 9 a 12 anos de idade?

Quais estudos demonstraramineficácia da vacina em mulheres não virgens? Idealmente, a vacina contra HPV deve ser administrada em mulheres antes da primeira relação sexual, nas quais se espera obter o maior benefício da vacina. No entanto, mulheres já infectadas por um tipo de HPV incluído na vacina serão protegidas contra doença causada por outros tipos constantes na vacina? ${ }^{7}$.

O câncer de colo de útero não é muitíssimo mais freqüente nos Estados Unidos da América (EUA) do que na Espanha. A incidência por 100.000 mulheres, ajustada por idade pela população mundial, é quase igual nos dois países: EUA - 7,7; Espanha - 7,6 ${ }^{8}$.
O conhecimento atual da história natural da infecção por HPV, apesar de lacunas importantes, indica que a vacinação profilática contra HPV 16 e 18 provavelmente reduzirá a incidência e a mortalidade por câncer de colo de útero. A vacina proporciona elevada proteção contra NIC 3, lesão reconhecidamente precursora do câncer cervical invasivo. Em estudo recente, o risco de câncer invasivo em mulheres com NIC 3 não tratadas foi 50 a 100 vezes o risco das mulheres tratadas ${ }^{9}$. Devemos aguardar décadas para comprovar a redução da incidência e mortalidade por esse câncer, para só então introduzir a vacinação?

A meu ver, o custo extremamente elevado representa o maior óbice para a introdução da vacina contra HPV, particularmente nos países em desenvolvimento.

Por último, desejo criticar o emprego do termo "promiscuidade". Diversos estudos mostram que proporção considerável (em alguns mais da metade) das mulheres com câncer de colo de útero refere apenas um parceiro sexual durante a vida ${ }^{10-12}$. Skrabanek ${ }^{13}$ menciona estudos que definem promiscuidade como relação sexual com mais de um, ou mais de dois parceiros. Segundo esse autor, parece que promiscuidade, se tiver algum significado, denota ter mais sexo que o investigador.

\section{Referências}

1. Bosch FX, Lorincz A, Muñoz N, Meijer CJLM, Shah KV. The causal relation between human papillomavirus and cervical cancer. J Clin Pathol 2002; 55: 244-65.

2. Schiffman M, Castle PE, Jeronimo J, Rodriguez AC, Wacholder S. Human papillomavirus and cervical cancer. Lancet 2007; 370: 890-907.

3. Muñoz N, Bosch FX, de Sanjosé S, Herrero R, Castellsagué X, Shah KV, et al. Epidemiologic classification of human papillomavirus types associated with cervical cancer. N Engl J Med 2003; 348: 51827.
4. Wright TC, Bosch FX, Franco EL, Cuzick J, Schiller JT, Garnett GP, et al. Chapter 30: HPV vaccines and screening in the prevention of cervical cancer; conclusions from a 2006 workshop of international experts. Vaccine 2006; 24(S3): S251-61.

5. Bosch FX, Castellsagué X, de Sanjosé S. HPV and cervical cancer: screening or vaccination? Brit J Cancer 2008; 98: 15-21.

6. Franco EL, Harper DM. Vaccination against human papillomavirus infection: a new paradigm in cervical cancer control. Vaccine 2005; 23: 2388-94. 
7. Markowitz LE, Dunne EF, Saraiya M, Lawson HW, Chesson H, Unger ER. Quadrivalent Human Papillomavirus Vaccine: Recommendations of the Advisory Committee on Immunization Practices (ACIP). MMWR Recomm Rep 2007; 56(RR2): $1-24$.

8. Ferlay J, Bray F, Pisani P, Parkin DM. GLOBOCAN 2002. Cancer Incidence, Mortality and Prevalence Worldwide. IARC CancerBase No. 5, version 2.0 IARCPress, Lyon, 2004.

9. McCredie MRE, Sharples KJ, Paul C, Baranyai J, Medley G, Jones RW, et al. Natural history of cervical neoplasia and risk of invasive cancer in women with cervical intraepithelial neoplasia 3: a retrospective cohort study. Lancet Oncol 2008; 9: 425-34.
10. Herrero R, Brinton LA, Reeves WC, Brenes MM, Tenorio F, de Debritton RC, et al. Sexual behavior, venereal diseases, hygiene practices, and invasive cervical cancer in a high-risk population. Cancer 1990; 65: 3806.

11. Bosch FX, Muñoz N, de Sanjosé S, Izarzugaza I, Gili M, Viladiu P, et al. Risk factors for cervical cancer in Colombia and Spain. Int J Cancer 1992; 52: 750-8.

12. Eluf-Neto J, Booth M, Muñoz N, Bosch FX, Meijer CJLM, Walboomers JMM. Human papillomavirus and invasive cervical cancer in Brazil. Brit J Cancer 1994; 69: 114-9.

13. Skrabanek P. Cervical cancer in nuns and prostitutes - A plea for scientific continence. J Clin Epidemiol 1988; 41: 577 82. 